\title{
A FACILE AND EFFICIENT SYNTHESIS OF HIGHLY FUNCTIONALIZED TERMINAL OLEFINES FROM $\alpha$-ALKOXY- $\beta$-HALIDES USING ZINC DUST ${ }^{\Psi}$
}

\author{
SAKKARAPALAYAM M. MAHALINGAM"A, HEMA KRISHNAN ${ }^{A}$ AND HARI N. PATI ${ }^{B, *}$ \\ ${ }^{A}$ Department of Chemistry, Indian Institute of Technology Madras, Chennai 600 036, India \\ ${ }^{B}$ Department of Chemistry, Sambalpur University, Jyoti Vihar 768 019, India \\ $\Psi$ Dedicated to Prof. V. S. Parmar, University of Delhi on his $60^{\text {th }}$ birthday. \\ (Received 8 July 2008 - Accepted 30 September 2008)
}

\begin{abstract}
A simple and efficient method of zinc dust/ammonium chloride system for the terminal olefination of $\alpha$-alkoxy- $\beta$-halides has been described. The reaction is carried out under mild conditions and yields of the corresponding terminal olefinic products are good. The significant feature of this method is the isolation of the pure product by simple work up in a short time.
\end{abstract}

Keywords: Terminal olefines / zinc dust / olefination / dehydrohalogenation.

\section{INTRODUCTION}

The terminal olefination or dehydrohalogenation of $\alpha$-alkoxy- $\beta$-halides is a useful chemical transformation in the synthesis of numerous organic compounds and also desired during the synthesis of compounds which are key intermediates in the synthesis of many pharmacological important substances. These terminal olefinic compounds are very important key precursors to synthesis the natural products via the ring closing metathesis ${ }^{1}$ and radical cyclization. ${ }^{2}$

Earlier reports reveal that the terminal olefination or dehydrohalogenation of $\alpha$-alkoxy- $\beta$-halides has been achieved with systems like $\mathrm{Cp}_{2} \mathrm{TiBH}_{4}$ and $\left[\mathrm{Cp}_{2} \mathrm{Ti}^{n} \mathrm{Bu}_{2}\right] \mathrm{MgCl}^{+}{ }^{3}$ However, these systems require reaction times as long as $5-10$ hours at reflux, expensive catalysts and also offer very low yields. Therefore, there is need of an efficient and inexpensive non toxic reagent system for the terminal olefination of $\alpha$-alkoxy- $\beta$-halides.

In order to develop new organic transformation, we report herein that zinc dust $/ \mathrm{NH}_{4} \mathrm{Cl}$ reagent system, might be a useful and inexpensive reagent for the reduction of $\alpha$-alkoxy- $\beta$-halides. Although, zinc has also been extensively used in the preparation of organometallic compounds ${ }^{4}$ and as a reducing agent $t^{5}$ in organic synthesis. The utility of zinc for the synthesis of $\beta, \alpha$-unsaturated ketones by a reaction of an acid chloride with allyl bromide ${ }^{6}$ and homoallylic alcohols $\mathrm{s}^{7}$ has been demonstrated. Furthermore, the zinc mediated amide formation, ${ }^{8}$ Fridel-Crafts acylation ${ }^{9}$ and carbamate formation ${ }^{10}$ has also been reported. In this context, the use of zinc as non-toxic 'green' reagent in organic synthetic processes has gained considerable importance due to its ability to promote and catalyze organic transformations of commercial importance under ambient conditions, without the need for any added catalyst or ligand.

\section{RESULTS AND DISCUSSION}

We began our study with the $\alpha$-alkoxy- $\beta$-halide 1a. ${ }^{11}$ Compound containing a neighbouring alkoxy group $(\mathrm{OBn})$ and a halo (iodo) group at $\beta$ position and reaction with zinc dust in ammonium chloride could conceivably give rise to either the acylic $\beta$-elimination product $\mathbf{2 a}$ or the simple reduced compound $\mathbf{3}$.

In this letter, we report a rapid and efficient reductive elimination of $\alpha$-alkoxy- $\beta$-halides to the corresponding terminal olefinic products using low

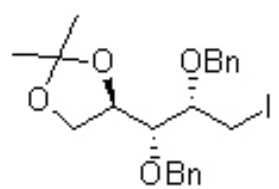

$1 \mathbf{a}$

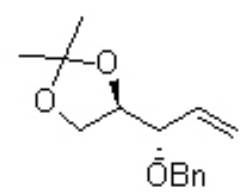

2a

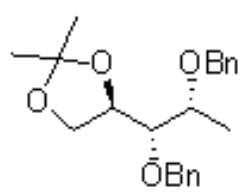

3
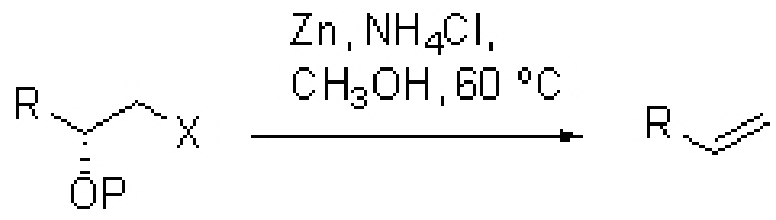

2

$$
\begin{aligned}
& \mathrm{P}=\mathrm{Bn} ; \mathrm{Me} \\
& \mathrm{X}=\mathrm{Br} ; \mathrm{l}
\end{aligned}
$$

1

\section{Scheme 1}

We found that the reductive elimination of 1a with zinc dust and ammonium chloride furnished only the $\beta$-elimination product $\mathbf{2 a}$ and not the simple reduced compound $\mathbf{3}$. The reaction was very clean with satisfactory yield and there was no side products observed.

The possible mechanism is depicted in figure 1. Probably, the $\alpha$-alkoxy$\beta$-halides react with zinc leads to the formation of either the intermediate $\mathbf{A}$ or B. These reactions are obviously substitution reactions, but they cannot be classified as nucleophilic substitutions. The facile elimination of the POZnX from the intermediate $\mathbf{A}$ or $\mathbf{B}$ furnished the terminal olefin $\mathbf{2}$. From these observations the $\alpha$-alkoxy- $\beta$-halides are considered to be masked olefins (Figure 1).

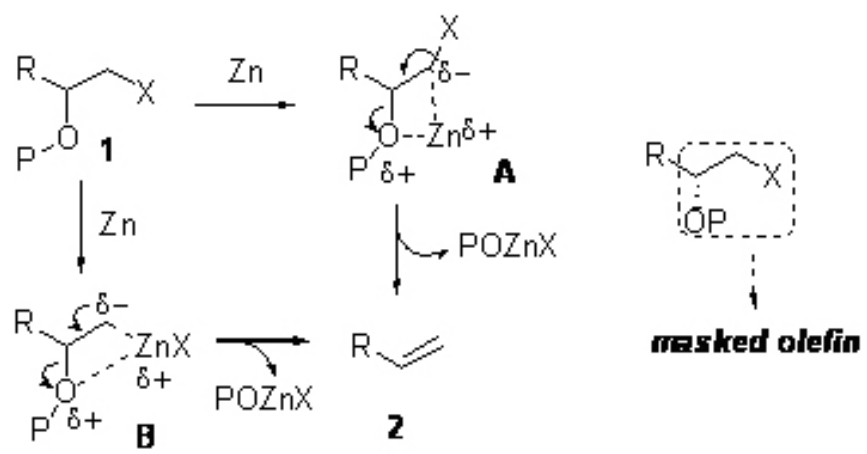

Figure 1.

cost zinc dust and ammonium chloride at $60{ }^{\circ} \mathrm{C}$ in methanol as depicted in Scheme 1.
The reaction on substrate 1a afforded compound $\mathbf{2 a}$, which was conformed by ${ }^{1} \mathrm{H}$ NMR and ${ }^{13} \mathrm{C}$ NMR spectrum. The one proton from the methine $(\mathrm{CH})$ unit of the olefinic residue appeared as multiplets from $\delta 5.77$ to $\delta 5.88 \mathrm{ppm}$ and the remaining two terminal olefinic protons $\left(\mathrm{CH}_{2}\right)$ appeared as multiplets from 
$\delta 5.31$ to $\delta 5.39 \mathrm{ppm}$. Firm evidence for the terminal olefination was obtained from peaks at $\delta 119.7$ (methylene; $\mathrm{CH}_{2}$ ) and $135.3 \mathrm{ppm}$ (methine; $\mathrm{CH}$ ) in the ${ }^{13} \mathrm{C}$ NMR spectrum. Also from the DEPT spectrum, one olefinic methylene carbon [at $\delta 119.7 \mathrm{ppm}$ ] and one olefinic methane carbon [at $\delta 135.3 \mathrm{ppm}$ ] were visible and it was clearly indicating the terminal olefinic product formation.

The reduction of $\alpha$-alkoxy- $\beta$-halides in the presence of zinc dust and ammonium chloride was completed within three to four hours (Table 1). The course of reaction was monitored by TLC. The work-up and isolation of the products were as usual. Thus, the $\alpha$-alkoxy- $\beta$-halides reduced by this system were obtained in good yields and no undesired side product was observed. Some of the results shown in table 1 clearly indicate the scope and generality of the reaction with respect to various $\alpha$-alkoxy- $\beta$-halides. All products were characterized by different spectroscopic techniques.

Compound $\mathbf{1 b}$ and $\mathbf{1 c}$ underwent reductive elimination, to give vinyl compounds $\mathbf{2 b}$ with good yields. We have also examined the four carbon $\alpha$-benzyloxy- $\beta$-halide $\mathbf{1 d}$ under the similar reaction conditions and as expected, we isolated the olefinic product $\mathbf{2 c}$ with $78 \%$ yield (Figure 2 ).

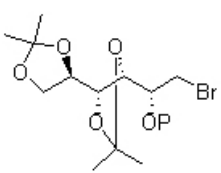

1b: $P=B n$

1e: $P=M e$

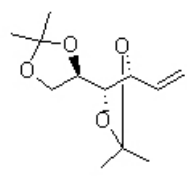

2b

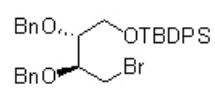

1d<smiles>C=C[C@@H](C[OH+][SbH2])O[SnH3]</smiles>

2e
Figure 2.

This similar kind of observation was noted by Hersant et. al. when using excess of $\mathrm{Cp}_{2} \mathrm{TiCl}$ as a reducing agent for $\alpha$-acetoxy- $\beta$-halides under photo irradiation conditions. ${ }^{3}$ But they observed a mixture of products like $\beta$-eliminated product and simple reduced compound. In comparison with $\mathrm{Cp}_{2} \mathrm{TiCl}$, zinc dust with ammonium chloride shows more reactivity, less reaction times and exclusive $\beta$-elimination product with higher yields (Table 1).

Table 1: Reductive elimination of $\alpha$-alkoxy- $\beta$-halides using zinc and $\mathrm{NH}_{4} \mathrm{Cl}$ in methanol

\begin{tabular}{ccccc}
\hline Entry & Substrate & Product & $\begin{array}{c}\text { Reaction } \\
\text { conditions } \\
\text { \& Time }\end{array}$ & Yield (\%) ${ }^{\mathrm{a}}$ \\
\hline 1 & $\mathbf{1 a}$ & $\mathbf{2 a}$ & $60^{\circ} \mathrm{C}, 3 \mathrm{~h}$ & 80 \\
2 & $\mathbf{1 b}$ & $\mathbf{2 b}$ & $60^{\circ} \mathrm{C}, 3 \mathrm{~h}$ & 82 \\
3 & $\mathbf{1 c}$ & $\mathbf{2 b}$ & $60^{\circ} \mathrm{C}, 4 \mathrm{~h}$ & 78 \\
4 & $\mathbf{1 d}$ & $\mathbf{2 c}$ & $60^{\circ} \mathrm{C}, 3.5 \mathrm{~h}$ & 76 \\
\hline
\end{tabular}

a'Yields refer to pure isolated products and were characterized by spectral data.

The starting substrate 1-deoxy-1-iodo-2,3-di- $O$-benzyl-4,5-Oisopropylidene-D-arabinitol (1a) was prepared from the corresponding standard literature method starting from D-(-)-arabinose. ${ }^{11}$ The substrates $2-O$ benzyl-1-bromo-3,4:5,6-di- $O$-isopropylidene-D-glucitol (1b) and 2- $O$-methyl1-bromo-3,4:5,6-di- $O$-isopropylidene-D-glucitol (1c) were conveniently prepared starting from D-gluconolactone (Scheme 2). The procedure starts with 1,2:3,4:5,6-tri- $O$-isopropylidene-D-gluconate (4), conveniently prepared on a multi-gram scale by the procedure of Jarosz et. al., in a single step from D-gluconolactone. ${ }^{12}$ Lactone $(\mathbf{4})$ on heating with morpholine in toluene at $90{ }^{\circ} \mathrm{C}$ furnished $\alpha$-hydroxy amide $\mathbf{5}$ in an isolated yield of $95 \%$. The $\alpha$-hydroxy amide 5 was subjected to $O$-benzylation/ $O$-methylation using sodium hydride as base in DMF at room temperature. Clean reaction was resulted in 30 minutes, giving the $\alpha$-O-benzyl $/ \alpha-O$-methyl amide (6) in an isolated yield of $90 \%$ after the usual work-up and silica-gel chromatography. The amide $\mathbf{6}$ underwent clean reduction with sodium borohydride in ethanol at $60{ }^{\circ} \mathrm{C}$, furnishing the 2-O-benzyl/2-O-methyl glucitol derivative 7. Bromination under modified Mitsunobu conditions using triphenyl phosphine and $N$-bromosuccinimide at $60{ }^{\circ} \mathrm{C}$ yielded the compound $\mathbf{1 b}$ and $\mathbf{1 c}$ (Scheme 2).
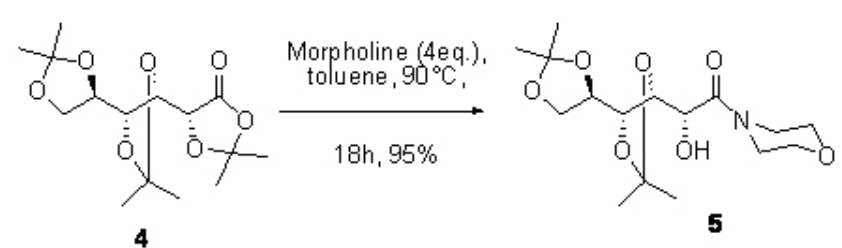

4

$\mathrm{NaH}, \mathrm{RX}$ DMF', $0^{\circ} \mathrm{C}-\mathrm{rt}$<smiles>[X]C[C@H](O)[C@@H](O)[C@@H](OC(C)(C)C)[C@H]1COC(C)(C)O1</smiles>

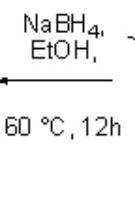<smiles>CC1COC(C)(C)O1</smiles>
$30 \mathrm{~min}$

$$
\left.\begin{array}{ll}
\text { 7a: } R=\mathrm{Bn} ; X=\mathrm{OH} \\
\text { 7b: } \mathrm{R}=\mathrm{Me} ; \mathrm{X}=\mathrm{OH}
\end{array}\right] \quad \begin{aligned}
& \mathrm{NBS}, \\
& \mathrm{PPh}_{3} \\
& \text { 1b: } \mathrm{R}=\mathrm{Bn} ; \mathrm{X}=\mathrm{Br} \\
& \text { 1e: } \mathrm{R}=\mathrm{Me} ; \mathrm{XMF}=\mathrm{Br}
\end{aligned} \quad \begin{aligned}
& 60^{\circ} \mathrm{C}
\end{aligned}
$$

NBS,

$\mathrm{PPh}_{3}$ DMF

$$
\begin{aligned}
& \text { 6a: } R=B n \\
& \text { 6b: } R=M e
\end{aligned}
$$

Scheme 2.

The four carbon $\beta$ - $O$-benzyl protected bromo compound $1 \mathbf{d}$ was prepared starting from D-(+)-tartaric acid (8). Stirring D-(+) tartaric acid with a catalytic amount of concentrated sulphuric acid in methanol at room temperature for 6 hours gave dimethyl tartrate $(\mathbf{9})$ in $80 \%$ yield. Di- $O$-benzylation of 9 using silver oxide and benzyl bromide furnished compound 10, followed by sodium borohydride reduction of $O$-benzyl protected $\alpha$-hydroxy methyl ester to afford the diol 11. For mono protection, the purified diol $\mathbf{1 1}$ was subjected to 1.0 equivalent of TBDPS-Cl in the presence of imidazole to afford the alcohol 12 in $72 \%$ yield. Bromination under modified Mitsunobu conditions using triphenyl phosphine and $\mathrm{N}$-bromosuccinimide at $60{ }^{\circ} \mathrm{C}$ yielded compound $1 \mathbf{d}$ in $78 \%$ (Scheme 3 ).<smiles>O=C(O)C(O)C(O)C(=O)O</smiles>

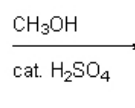

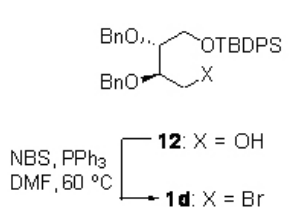

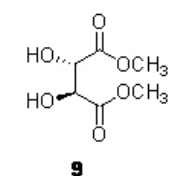

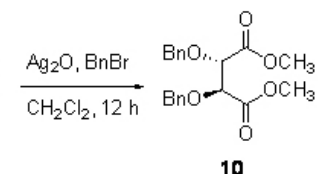

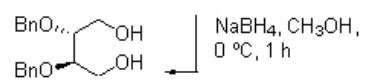

11
Scheme 3 .

In general, the terminal olefination reactions (Table 1) are very clean, reasonably fast and high yielding compare to the other reported procedures. The present procedure using zinc dust with ammonium chloride provides an efficient route to highly functionalized terminal olefines.

\section{CONCLUSIONS}

In conclusion, we have demonstrated a very simple, efficient and practical method for the terminal olefination using zinc dust with ammonium chloride. The important features of this method include: (a) operational simplicity, (b) no need for any other additive to promote the reaction, (c) shorter reaction time, (d) the use of cheap, commercially available, non toxic reagents, and (e) good to moderate yields of desired products. Moreover, 14 new compounds have been synthesized and their data cited in this report for the first time. 


\section{EXPERIMENTAL SECTION}

\section{General information}

${ }^{1} \mathrm{H}$ NMR spectra were recorded on $400 \mathrm{MHz}$ Bruker AVANCE 400 spectrometer and ${ }^{13} \mathrm{C}$ NMR spectra were recorded on $100 \mathrm{MHz}$ Bruker AVANCE 400 spectrometer, respectively, using $\mathrm{CDCl}_{3}$ as solvent and TMS as an internal standard. IR spectra were recorded on a Perkin-Elmer FT/IR 100 spectrometer. Mass spectra were recorded on Agilent-1100 mass spectrometer. Optical rotations were measured by a Rudolph Autopol V polarimeter. All the reactions were monitored by thin layer chromatography (TLC). TLC was performed on $\mathrm{F}_{254}, 0.25 \mathrm{~mm}$ silica gel plates (Merck). Plates were eluted with appropriate solvent systems, and then stained with either alkali $\mathrm{KMnO}_{4}$ or Ceric ammonium molybdate solutions prepared in the laboratory. The developed plates were first analysed under UV $254 \mathrm{~nm}$ then stained with appropriate reagent. Column chromatography was performed using silica gel with particle size 100-200 mesh

\section{General procedure for $\boldsymbol{O}$-alkylation using $\mathrm{NaH}$.}

A solution of the hydroxy compound $(1 \mathrm{mmol})$ in dry DMF $(6 \mathrm{ml})$ was added to oil-free sodium hydride $(1.1 \mathrm{mmol})$ in DMF $(1 \mathrm{ml})$ at $0{ }^{\circ} \mathrm{C}$ under inert atmosphere. This was followed by addition of benzyl bromide/methyl iodide $(1.1 \mathrm{mmol})$; the reaction mixture was allowed to stir at room temperature for 30 minutes. On completion of the reaction as monitored by thin layer chromatography, the product was extracted using ethyl acetate $(20 \mathrm{ml})$ and the DMF was removed by washing with brine solution $(2 \times 50 \mathrm{ml})$. The organic layer was separated and evaporated under reduced pressure to yield the $O$-alkylated product. The crude product was subjected to silica gel column chromatography (ethyl acetate: hexane, 2: 8) to give a pure compound.

\section{Morpholino - (3,4:5,6-di- $O$-isopropylidene) -D - gluconamide (5):}

To a solution of the triacetonide $4(5 \mathrm{~g}, 5.8 \mathrm{mmol})$ in toluene $(16 \mathrm{ml})$, morpholine $(5.5 \mathrm{~mL}, 63.3 \mathrm{mmol})$ was added and the reaction mixture was stirred at $90{ }^{\circ} \mathrm{C}$ for 18 hours. On completion of the reaction as monitored by thin layer chromatography, the reaction mixture was allowed to cool and toluene was evaporated under reduced pressure. Morpholine was removed by addition of toluene $(2 \times 10 \mathrm{ml})$ and distillation of the azeotropic mixture under reduced pressure. The crude product was subjected to silica gel column chromatography (ethyl acetate: hexane, 2: 8) to yield the pure compound 5 as a white solid. $[\alpha]_{D}^{27}:-15.8^{\circ}\left(\mathrm{c} 1, \mathrm{CHCl}_{3}\right)$. IR (neat) $v_{\max } \mathrm{cm}^{-1:} 3430,1650$, 1371, 1247, 1213, 1112, 1063, 847, 511. ${ }^{1} \mathrm{H}$ NMR: $\delta 1.26,1.31,1.34,1.37$ (4 $\left.\mathrm{x} \mathrm{s}, 12 \mathrm{H}, 4 \mathrm{x} \mathrm{CH}_{3}\right) ; 3.37-3.72(\mathrm{~m}, 8 \mathrm{H}$, morpholino); $3.76(\mathrm{~d}, J=9.2 \mathrm{~Hz}, 1 \mathrm{H}$, $-\mathrm{OH}) ; 3.87-3.94(\mathrm{~m}, 2 \mathrm{H}, \mathrm{CH} C \mathrm{CH}) ; 3.95-4.14(\mathrm{~m}, 3 \mathrm{H}, 3 \times \mathrm{CH}) ; 4.51(\mathrm{~d}, J=9.2$ $\mathrm{Hz}, 1 \mathrm{H}, \mathrm{CHCO}) ;{ }^{13} \mathrm{C}$ NMR: $\delta$ 26.6, 27.2, $\left(4 \mathrm{x} \mathrm{CH}_{3}\right) ; 42.9,45.4\left(2 \times \mathrm{N}_{-} \mathrm{CH}_{2}\right) ; 66.3$ $\left(\mathrm{CH}_{2} \mathrm{CH}\right) ; 66.7\left(2 \times \mathrm{O}_{-} \mathrm{CH}_{2}\right) ; 68.0\left(\mathrm{CH}_{2} \mathrm{CH}\right) ; 77.1,77.6(2 \times \mathrm{CH}) ; 80.5(\mathrm{CHCO})$; 109.6, 110.6, $\left(2 \times \mathrm{CMe}_{2}\right) ; 170.3(\mathrm{CO}) ; \mathrm{HRMS}(\mathrm{TOF} \mathrm{MS} \mathrm{ES}+) \mathrm{m} / \mathrm{z}[\mathrm{M}+\mathrm{H}]$ calcd. for $\mathrm{C}_{16} \mathrm{H}_{27} \mathrm{NO}_{7} 345.1866$, found 345.1870 .

1-Morpholino-(2-O-benzyl-3,4:5,6-di- $O$-isopropylidene)-Dgluconamide (6a):

This compound was obtained as colourless crystals in $90 \%$ yield. $[\alpha]_{D}{ }^{27}$ : $+10.79^{\circ}(\mathrm{c} 1, \mathrm{CHCl})$. IR (neat) $v \mathrm{~cm}^{-1}: 1637,1456,1371,1252,1114,1070$, 847, 738, 699, 584. ${ }^{1} \mathrm{H}$ NMR: $\delta 1.25,1.29\left(2 \mathrm{x} \mathrm{s}, 12 \mathrm{H}, 4 \times \mathrm{CH}_{3}\right) ; 3.40-3.70(\mathrm{~m}$, $8 \mathrm{H}$, morpholino); 3.78-3.88 (m, 2H, $\left.\mathrm{CH}_{2} \mathrm{CH}\right) ; 3.97-4.20$ (m, 3H, $\left.3 \times \mathrm{CH}\right) ; 4.31$ $(\mathrm{d}, J=2.5 \mathrm{~Hz}, 1 \mathrm{H}, \mathrm{CHCO}) ; 4.42\left(\mathrm{~d}, J=11.5 \mathrm{~Hz}, 1 \mathrm{H}, \mathrm{PhCH}_{\mathrm{a}} \mathrm{H}_{\mathrm{b}}\right) ; 4.59(\mathrm{~d}, J=$ $\left.11.5 \mathrm{~Hz}, 1 \mathrm{H}, \mathrm{PhCH}_{\mathrm{H}} H_{\mathrm{b}}\right) ; 7.21-7.32(\mathrm{~m}, 5 \mathrm{H}, \mathrm{Ar}-\mathrm{H}) ;{ }^{13} \mathrm{C}$ NMR: $\delta$ 2 25.2, 26.6, 27.2 (4x CH$) ; 43.2,45.9$ (2 x N-CH $)$; 66.9, 67.1 (2 x O-CH , morpholinyl); 67.6 $\left(\mathrm{CH}_{2} \mathrm{CH}\right) ; 72.9\left(\mathrm{CH}_{2} \mathrm{CH}\right) ; 76.94,76.96(2 \times \mathrm{CH}) ; 77.4\left(\mathrm{PhCH}_{2}\right) ; 80.5(\mathrm{CHCO})$; 109.7, $109.9\left(2 \times \mathrm{CMe}_{2}\right) ; 127.8,128.1,128.5,136.9$ (Aromatic); $168.5(\mathrm{CO})$ ; HRMS (TOF MS ES+) m/z [M+H] $]^{+}$calcd. for $\mathrm{C}_{23} \mathrm{H}_{33} \mathrm{NO}_{7} 436.2335$, found 436.2340

1-Morpholino-(2-O-methyl-3,4:5,6-di- $O$-isopropylidene)-Dgluconamide (6b):

This compound was obtained as a syrupy liquid. $[\alpha]_{D}^{27}:+15.6^{\circ}$ (c 1 , $\mathrm{CHCl}_{3}$ ). IR (neat) $v \mathrm{~cm}^{-1}: 1640,1455,1370,1075 .{ }^{1} \mathrm{H}$ NMR: $\delta 1.34,1.36$, $1.37,1.41\left(4 \times \mathrm{x} \mathrm{s}, 12 \mathrm{H}, 4 \times \mathrm{CH}_{3}\right) ; 3.42\left(\mathrm{~s}, 3 \mathrm{H}, \mathrm{H}-\mathrm{OCH}_{3}\right), 3.60-3.92(\mathrm{~m}, 8 \mathrm{H}$, morpholino); 3.94-3.40 (m, 1H), 4.00-4.23 (m, 5H); ${ }^{13} \mathrm{C}$ NMR: $\delta 25.1,26.5$, 26.6, $27.1\left(4 \mathrm{x} \mathrm{CH}_{3}\right) ; 43.2$, $45.6\left(2 \mathrm{x} \mathrm{N}^{\left.-\mathrm{CH}_{2}\right)}\right)$ 58.1 $\left(\mathrm{OCH}_{3}\right) ; 67.0,67.1(2 \mathrm{x}$ $\mathrm{O}-\mathrm{CH}_{2}$, morpholinyl); $67.6\left(\mathrm{CH}_{2} \mathrm{CH}\right) ; 76.6\left(\mathrm{CH}_{2} \mathrm{CH}\right) ; 77.0,70.6(2 \times \mathrm{CH}) ; 83.5$ (CHCO); 109.6, 109.7 ( $\left.2 \times \mathrm{CMe}_{2}\right) ; 168.3$ (CO) ; HRMS (TOF MS ES+) m/z $[\mathrm{M}+\mathrm{H}]^{+}$calcd. for $\mathrm{C}_{17} \mathrm{H}_{29} \mathrm{NO}_{7} 360.2022$, found 360.2022 .
General Procedure for $\mathrm{NaBH}_{4}$ reduction.

To a solution of the starting substrate $(1 \mathrm{mmol})$ in $10 \mathrm{ml}$ of ethanol, sodium borohydride $(4 \mathrm{mmol})$ was added and the reaction mixture was stirred at $60{ }^{\circ} \mathrm{C}$ for 12 hours. On completion of the reaction as monitored by thin layer chromatography, the reaction mixture was allowed to cool and ethanol was evaporated under reduced pressure. The residue was dissolved in $25 \mathrm{ml}$ of water and the solution was neutralized using acetic acid. The product was extracted using diethyl ether $(2 \times 30 \mathrm{ml})$ and the combined ether extract was evaporated under reduced pressure to give the crude product. The crude product was subjected to silica gel column chromatography (ethyl acetate: hexane, 2.5: 7.5 ) to give pure compound.

2-O-Benzyl-3,4:5,6-di- $O$-isopropylidene-D-glucitol (7a):

This compound was obtained as colourless syrup. $[\alpha]_{\mathrm{D}}{ }^{27}:+26.38^{\circ}$ (c 1 , $\mathrm{CHCl}_{3}$ ). IR (neat) $v \quad \mathrm{~cm}^{-1}: 3481,1217,1071,772,698,669 .{ }^{1} \mathrm{H}$ NMR: $\delta$ $1.35,1.39,1.42\left(3 \mathrm{x} \mathrm{s}, 12 \mathrm{H}, 4 \times \mathrm{CH}_{3}\right) ; 3.60-4.20$ (multiplets, $\left.8 \mathrm{H}\right), 4.68(\mathrm{~d}$, $\left.J=11.7 \mathrm{~Hz}, 1 \mathrm{H}, \mathrm{PhCH} \mathrm{H}_{b}\right) ; 4.78\left(\mathrm{~d}, J=11.7 \mathrm{~Hz}, 1 \mathrm{H}, \mathrm{PhCH} H_{b}\right) ; 7.25-7.39$ (m, 5H, Ar-H); ${ }^{13} \mathrm{C}$ NMR: $\delta$ 25.2, 26.4, 26.7, $27.2\left(4 \mathrm{x} \mathrm{CH}_{3}\right) ; 62.2\left(\mathrm{CH}_{2} \mathrm{OH}\right)$; $67.9\left(\mathrm{C}-\mathrm{O}-\mathrm{CH}_{2}\right) ; 72.5\left(\mathrm{C}-\mathrm{O}-\mathrm{CH}_{2} \mathrm{CH}\right) ; 77.4,77.9(2 \times \mathrm{CH}) ; 78.1\left(\mathrm{PhCH}_{2}\right) ; 81.9$ $\left(\mathrm{CHCH}_{2} \mathrm{OH}\right) ; 109.77,109.79$ (2 x $\left.\mathrm{CMe}_{2}\right) ; 127.7,127.8,127.9,128.5,138.3$ (aromatic); HRMS (TOF MS ES+) m/z [M+Na] $]^{+}$calcd. for $\mathrm{C}_{19} \mathrm{H}_{28} \mathrm{O}_{6} 375.1784$, found 375.1789 .

2-O-Methyl-3,4:5,6-di- $O$-isopropylidene-D-glucitol (7b):

This compound was obtained as colourless syrup. $[\alpha]_{\mathrm{D}}^{27}:+23.40^{\circ}$ (c 1 , $\mathrm{CHCl}_{3}$ ). IR (neat) $v_{\mathrm{cm}^{-1}}: 1210,1075,772,698,668 .{ }^{1} \mathrm{H}$ NMR: $\delta 1.36,1.38$, $1.42,1.43\left(4 \mathrm{x} \mathrm{s}, 12 \mathrm{H}, 4 \times \mathrm{CH}_{3}\right) ; 3.38-3.42(\mathrm{~m}, 1 \mathrm{H}), 3.51\left(\mathrm{~s}, 3 \mathrm{H}, \mathrm{H}-\mathrm{OCH}_{3}\right)$, $3.75-3.80(\mathrm{~m}, 1 \mathrm{H}), 3.87-3.92(\mathrm{~m}, 1 \mathrm{H}), 3.95-4.02(\mathrm{~m}, 2 \mathrm{H}), 4.03-4.12(\mathrm{~m}, 2 \mathrm{H})$, 4.16-4.20 (m, 1H). ${ }^{13} \mathrm{C}$ NMR: $\delta$ 25.2, 26.4, 26.7, $27.1\left(4 \mathrm{x} \mathrm{CH}_{3}\right) ; 52.1,58.4$ $\left(\mathrm{OCH}_{3}\right), 61.5\left(\mathrm{CH}_{2} \mathrm{OH}\right) ; 65.8,68.0,80.1,81.9,109.7,109.9\left(2 \mathrm{x} \mathrm{CMe}_{2}\right)$.

General procedure for bromination using $\boldsymbol{N}$-bromosuccinimide.

To a solution of the hydroxy compound $(1 \mathrm{mmol})$ in dry DMF $(10 \mathrm{ml})$ was added triphenyl phosphine $(2 \mathrm{mmol})$ and $\mathrm{N}$-bromosuccinimide $(2 \mathrm{mmol})$. The reaction mixture was then heated at $60{ }^{\circ} \mathrm{C}$ for 3 hours. On completion of the reaction, water $(20 \mathrm{ml})$ was added and the mixture was extracted with diethyl ether $(3 \times 10 \mathrm{ml})$. The combined organic layer was dried over anhydrous sodium sulphate, and evaporated under reduced pressure to afford the crude residue. The residue was purified by silica-gel column chromatography $(8: 2$, hexane-ethyl acetate) to produce the pure bromo compound.

\section{2-O-Benzyl-1-bromo-3,4:5,6-di- $O$-isopropylidene-D-glucitol (1b):}

This compound was obtained as colourless syrup. IR (neat) $v \quad \mathrm{~cm}^{-1}: 1215$, 1071, 772, 690. ${ }^{1} \mathrm{H}$ NMR: $\delta 1.27,1.30,1.31,1.33\left(4 \mathrm{x} \mathrm{s}, 12 \mathrm{H}, 4 \mathrm{x} \mathrm{CH}_{3}\right) ; 3.47-$ $3.51(\mathrm{~m}, 2 \mathrm{H}), 3.70-3.76(\mathrm{~m}, 1 \mathrm{H}), 3.78-3.84(\mathrm{~m}, 1 \mathrm{H}), 3.87-3.93(\mathrm{~m}, 1 \mathrm{H}), 3.96-$ $4.03(\mathrm{~m}, 1 \mathrm{H}), 4.04-4.10(\mathrm{~m}, 1 \mathrm{H}), 4.15-4.20(\mathrm{~m}, 1 \mathrm{H}), 4.55(\mathrm{~d}, J=11.7 \mathrm{~Hz}, 1 \mathrm{H}$, $\left.\mathrm{PhCH}_{a} \mathrm{H}_{\mathrm{b}}\right) ; 4.72\left(\mathrm{~d}, J=11.7 \mathrm{~Hz}, 1 \mathrm{H}, \mathrm{PhCH}_{\mathrm{a}} H_{b}\right) ; 7.21-7.30(\mathrm{~m}, 5 \mathrm{H}, \mathrm{Ar}-\mathrm{H}) ;{ }^{13} \mathrm{C}$ NMR: $\delta$ 25.2, 26.6, 26.7, $27.2\left(4 \mathrm{x} \mathrm{CH}_{3}\right) ; 30.17\left(\mathrm{CH}_{2} \mathrm{Br}\right) ; 67.9\left(\mathrm{C}-\mathrm{O}-\mathrm{CH}_{2}\right) ; 73.5$ $\left(\mathrm{C}-\mathrm{O}-\mathrm{CH}_{2} \mathrm{CH}\right) ; 77.1,77.3(2 \times \mathrm{CH}) ; 78.4\left(\mathrm{PhCH}_{2}\right) ; 80.1\left(\mathrm{CHCH}_{2} \mathrm{Br}\right) ; 109.7$, $109.8\left(2 \times \mathrm{CMe}_{2}\right) ; 127.8,127.9,128.2,137.9$ (Aromatic).

2-O-Methyl-1-bromo-3,4:5,6-di- $O$-isopropylidene-D-glucitol (1c):

This compound was obtained as colourless syrup. IR (neat) $v_{\max } \mathrm{cm}^{-1}$ : $1215,1071,772,690 .{ }^{1} \mathrm{H}$ NMR: $\delta 1.28,1.29,1.32,1.35(4 \times \mathrm{x} \mathrm{s}, 12 \mathrm{H}, 4 \mathrm{x}$ $\left.\mathrm{CH}_{3}\right)$; 3.45-3.50 (m, $\left.6 \mathrm{H}\right), 3.82-3.90(\mathrm{~m}, 2 \mathrm{H}), 3.95-4.03(\mathrm{~m}, 1 \mathrm{H}), 4.07-4.17(\mathrm{~m}$, $2 \mathrm{H}) ;{ }^{13} \mathrm{C}$ NMR: $\delta 21.65,25.63,26.05\left(4 \mathrm{x} \mathrm{CH}_{3}\right), 29.48\left(\mathrm{CH}_{2} \mathrm{Br}\right), 58.38\left(\mathrm{OCH}_{3}\right)$, $66.95\left(\mathrm{C}-\mathrm{O}-\mathrm{CH}_{2}\right), 76.16,78.81(2 \times \mathrm{CH}), 79.09\left(\mathrm{CHCH}_{2} \mathrm{Br}\right), 108.54,108.69$ $\left(2 \times \mathrm{CMe}_{2}\right)$

Synthesis of $(2 R, 3 R)$-dimethyl 2,3-bis $(O$-benzyl)tartrate $(10)$ :

A suspension of (+)-dimethyl tartrate (9) $(0.5 \mathrm{~g}, 2.8 \mathrm{mmol})$ in dichloromethane $(10 \mathrm{ml})$ was treated with silver oxide $(1.43 \mathrm{~g}, 6.2 \mathrm{mmol})$, and allowed to stir for 12 hours at room temperature. The reaction mixture was filtered through a celite bed, and washed with additional volumes of dichloromethane. The original filtrate and the dichloromethane washings were combined and evaporated to dryness on the rotary evaporator. The residue was subjected to silica gel chromatography to yield $(0.72 \mathrm{~g} ; 75 \%)$ pure $\mathbf{1 0}$ as colourless syrup. ${ }^{1} \mathrm{H}$ NMR: $\delta 3.65\left(6 \mathrm{H}, \mathrm{s}, \mathrm{H}-\left(2 \times \mathrm{CH}_{3}\right)\right), 4.38-4.44(4 \mathrm{H}, \mathrm{m})$, 4.85-4.88 (2H, m), 7.25-7.36 (10H, m, H-Ph). ${ }^{13} \mathrm{C}$ NMR: $\delta 52.1\left(\mathrm{OCH}_{3}\right), 73.2$ $\left(\mathrm{CH}_{2}(\right.$ benzylic) $), 78.2(\mathrm{CH}), 126.9,127.6,128.0,128.3,136.8(\mathrm{C}-\mathrm{Ph}), 169.5$ $(\mathrm{C}=\mathrm{O})$. 
$(2 R, 3 R)-2,3-B i s(b e n z y l o x y) b u t a n e-1,4-d i o l ~(11):$

This compound was obtained as colourless syrup in $77 \%$ yield. ${ }^{1} \mathrm{H}$ NMR: $\delta$ 3.68-3.83 (6H, m), 4.63-4.67 (4H, m), 7.25-7.36 (10H, m). ${ }^{13} \mathrm{C}$ NMR: $\delta 60.8$ $\left(\mathrm{CH}_{2}-\mathrm{OH}\right), 72.6\left(\mathrm{CH}_{2}\right.$ (benzylic) $), 78.9(\mathrm{CH}), 126.9,127.9,128.0,128.5,138.0$ (C-Ph).

Synthesis of (2R,3R)-2,3-bis(benzyloxy)-4-(tert-butyldiphenylsilyloxy) butan-1-ol (12):

To a solution of the dihydroxy compound $\mathbf{1 1}(0.5 \mathrm{~g}, 1.65 \mathrm{mmol})$ in dry DMF $(4 \mathrm{ml})$ was added imidazole $(0.224 \mathrm{~g}, 3.3 \mathrm{mmol})$ followed by chloro tert-butyl diphenylsilane $(0.5 \mathrm{~g}, 1.8 \mathrm{mmol})$ at room temperature. After 6 hours stirring, water $(15 \mathrm{ml})$ was added and the reaction mixture was extracted with ether $(3 \times 10 \mathrm{ml})$. The combined organic layer was dried over $\mathrm{Na}_{2} \mathrm{SO}_{4}$ and concentrated under reduced pressure. The residue thus obtained was purified by silica-gel column chromatography ( $8: 2$, hexane: ethyl acetate) to produce pure compound 12 as colourless syrup (0.48 g; 74\%). ${ }^{1} \mathrm{H}$ NMR: $\delta 1.05(9 \mathrm{H}, \mathrm{s}), 3.63-$ $3.70(2 \mathrm{H}, \mathrm{m}), 3.70-3.80(2 \mathrm{H}, \mathrm{m}), 3.82-3.87(2 \mathrm{H}, \mathrm{m}), 4.47-4.70(4 \mathrm{H}, \mathrm{m}), 7.25$ $7.43(16 \mathrm{H}, \mathrm{m}), 7.66-7.70(4 \mathrm{H}, \mathrm{m}) .{ }^{13} \mathrm{C}$ NMR: $\delta 20.33,27.9,62.9,64.0,66.5$, 73.9, 74.1, 80.2, 81.1, 128.1, 128.8, 128.9, 128.9, 129.0, 129.1, 129.5, 129.5, 129.7, 130.9, 130.9, 134.2, 134.3, 136.7, 136.8, 139.4, 139.4. HRMS (TOF MS ES+) $\mathrm{m} / \mathrm{z}[\mathrm{M}+\mathrm{Na}]^{+}$calcd. for $\mathrm{C}_{34} \mathrm{H}_{40} \mathrm{O}_{4} \mathrm{Si} 563.2594$, found 563.2590 .

$((2 R, 3 R)-2,3-B$ is (benzyloxy)-4-bromobutoxy)(tert-butyl) diphenylsilane(1d):

This compound was obtained as colourless syrup in $72 \%$ yield. ${ }^{1} \mathrm{H}$ NMR: $\delta 1.04(6 \mathrm{H}, \mathrm{s}), 1.57(3 \mathrm{H}, \mathrm{s}), 3.43-3.45(1 \mathrm{H}, \mathrm{m}), 3.50-3.58(1 \mathrm{H}, \mathrm{m}), 3.73-3.85$ $(2 \mathrm{H}, \mathrm{m}), 3.87-4.0(2 \mathrm{H}, \mathrm{m}), 4.55-4.75(4 \mathrm{H}, \mathrm{m}), 7.25-7.37(16 \mathrm{H}, \mathrm{m}), 7.63-7.669$ $(4 \mathrm{H}, \mathrm{m}) .{ }^{13} \mathrm{C}$ NMR: $\delta 19.0,26.8,30.0,31.3,62.6,73.3,73.7,78.9,127.7,127.7$, 127.7, 128.0, 128.1, 128.3, 128.3, 128.5, 129.7, 129.8, 135.6. HRMS (TOF MS $\mathrm{ES}+) \mathrm{m} / \mathrm{z}[\mathrm{M}+\mathrm{Na}]^{+}$calcd. for $\mathrm{C}_{34} \mathrm{H}_{39} \mathrm{O}_{3} \mathrm{SiBr} 625.1750$, found 625.1777 .

General procedure for the reductive elimination and debromination of $\alpha$-alkoxy- $\beta$-halides.

To a solution of the starting substrates 1a-d $(1 \mathrm{mmol})$ in methanol (10 $\mathrm{ml}$ ) was added ammonium chloride $(0.5 \mathrm{mmol})$ and zinc dust $(2 \mathrm{mmol})$. The mixture was stirred at $60^{\circ} \mathrm{C}$ for $3-4$ hours. After the completion of the reaction (monitored by TLC), the reaction mixture was filtered through celite. The filtrate was evaporated under vacuum and the residue was taken into chloroform or ether, washed twice with saturated brine solution and finally with water. The organic layer was dried over anhydrous sodium sulphate, and evaporation of the organic layer was followed by purification by column chromatography to yield the desired product.

Spectroscopic data for final olefinic products (2a-c):

Compound 2a. Colorless oil, IR (neat) $v_{\text {max }} \mathrm{cm}^{-1}: 3064,3032,2986,2932$, $2874,1655,1630$. ${ }^{1} \mathrm{H}$ NMR: $\delta 1.34(\mathrm{~s}, 3 \mathrm{H}), 1.40(\mathrm{~s}, 3 \mathrm{H}), 3.71-3.76(\mathrm{~m}, 1 \mathrm{H})$, $3.84-3.90(\mathrm{~m}, 1 \mathrm{H}), 4.00-4.15(\mathrm{~m}, 2 \mathrm{H}), 4.39(\mathrm{~d}, 1 \mathrm{H}, J=11.8 \mathrm{~Hz}), 4.63(\mathrm{~d}, 1 \mathrm{H}, J$ $=11.8,), 5.31-5.39(\mathrm{~m}, 2 \mathrm{H}), 5.76-5.88(\mathrm{~m}, 1 \mathrm{H}), 7.27-7.34(\mathrm{~m}, 5 \mathrm{H}) .{ }^{13} \mathrm{C} \mathrm{NMR}$ : $\delta 25.3,26.6,66.8,70.6,77.7,81.1,109.5,119.6,127.7,127.9,128.4,135.3$, 138.2; HRMS Calcd. for $\mathrm{C}_{15} \mathrm{H}_{20} \mathrm{O}_{3}\left([\mathrm{M}+\mathrm{Na}]^{+}\right) \mathrm{m} / z$ 249.1413, found 249.1415 .

Compound 2b. Colorless oil, IR (neat) $v_{\max } \mathrm{cm}^{-1}: 3089,2988,2935,2884$, 1647, 1457. ${ }^{1} \mathrm{H}$ NMR: $\delta 1.34(\mathrm{~s}, 3 \mathrm{H}), 1.41(\mathrm{~s}, 9 \mathrm{H}), 3.68-3.74(\mathrm{~m}, 1 \mathrm{H}), 3.93-3.96$ $(\mathrm{m}, 1 \mathrm{H}), 4.08-4.16(\mathrm{~m}, 2 \mathrm{H}), 4.34-4.37(\mathrm{~m}, 1 \mathrm{H}), 5.21(\mathrm{~d}, 1 \mathrm{H}, J=11.9 \mathrm{~Hz}), 5.41$ $(\mathrm{d}, 1 \mathrm{H}, J=11.9 \mathrm{~Hz}), 5.87-6.00(\mathrm{~m}, 1 \mathrm{H}) .{ }^{13} \mathrm{C}$ NMR: $\delta 25.2,26.7,26.9,27.0$, 67.0, 76.7, 80.5, 81.2, 109.4, 109.6, 117.2, 135.9. HRMS Calcd. for $\mathrm{C}_{12} \mathrm{H}_{20} \mathrm{O}_{4}$ $\left([\mathrm{M}+\mathrm{Na}]^{+}\right) \mathrm{m} / \mathrm{z} 251.1259$, found 251.1252 .

Compound 2c. Colorless oil, IR (neat) $v_{\max } \mathrm{cm}^{-1}: 3090,2985,2935,2884$ ${ }^{1} \mathrm{H}$ NMR: $\delta 1.05(9 \mathrm{H}, \mathrm{s}), 3.90-3.94\left(\mathrm{~m}, 1 \mathrm{H}, \mathrm{H}-\mathrm{OCH}_{2}\right), 4.13-4.16(\mathrm{~m}, 2 \mathrm{H}$, $\mathrm{H}-\mathrm{OCH}_{2} \&$ H-olefinic), 4.38-4.39 (m, 1H, H-olefinic), 4.48-4.51(t, $1 \mathrm{H}, J=6.7$ $\mathrm{Hz}, \mathrm{H}-\mathrm{OCH}$ ), 4.79-4.88 (dd, $2 \mathrm{H}, J=11.9 \mathrm{~Hz}, \mathrm{H}_{-} \mathrm{CH}_{2}$ (benzylic)), 7.25-7.43 $(11 \mathrm{H}, \mathrm{m}), 7.66-7.70(4 \mathrm{H}, \mathrm{m}) \cdot{ }^{13} \mathrm{C}$ NMR: $\delta 20.3(\mathrm{C}-\mathrm{Si}), 27.9\left(\mathrm{Si}\left(\mathrm{CH}_{3}\right)_{2}\right), 62.9$, $68.3\left(\mathrm{OCH}_{2}\right.$-benzylic), 116.5 ( $\mathrm{CH}_{2}$-olefinic), 128.1, 128.8, 128.9, 128.9, 129.0, $129.1,129.5,129.5,129.7,130.9,130.9,134.7$ (CH- olefinic), 134.3, 136.7, $136.8,139.4,139.4$ (C-Ar).

\section{ACKNOWLEDGEMENTS}

The authors thank the Department of Chemistry, IIT-Madras and Sambalpur University for support to the research.

\section{REFERENCES}

1. (a) K. R. Buszek, N. Sato, Y. Jeong, Tetrahedron Lett., 43, 181, (2002).(b) N. Brown, B. Xie, N. Markina, D. VanderVelde, Jean-Pierre H. Perchellet, E. M. Perchellet, K. R. Crow, K. R. Buszek, Bioorg. Med. Chem. Lett. 18, 4876,(2008).

2. (a) A. F. Barrero, M. Quilez del, F. Jose F, E. M. Sanchez, J. F. Arteaga, Eur. J. Org. Chem. 1627, (2006). (b) K. M. Sang, W. S. Kook, S. W. Na, E. Lee, Angew. Chem, Int. Ed. Engl. 9, 1733, (2008).

3. G. Hersant, M. B. Ferjani, S. M. Bennett, Tetrahedron Lett., 45, 8123, (2004).

4. (a) P. Knochel, R. D. Singer, R. D. Chem. Rev. 93, 2117, (1993). (b) E. Negishi, Organometallics in Organic Synthesis; Wiley: New York, 1980.

5. M. Hudlicky, Reductions in Organic Synthesis; Wiley: New York, 1984.

6. B. C. Ranu, A. Majee, A. R. Das, Tetrahedron Lett., 37, 1109, (1996).

7. B. C. Ranu, A. Majee, A. R. Das, Tetrahedron Lett., 36, 4885, (1995).

8. H. M. Meshram, G. S. Reddy, M. M. Reddy, J. S. Yadav, Tetrahedron Lett., 39, 4103, (1998).

9. J. S. Yadav, G. S. Reddy, M. M. Reddy, H. M. Meshram, Synth. Commun., 28, 2203, (1999).

10. J. S. Yadav, G. S. Reddy, M. M. Reddy, H. M. Meshram, Tetrahedron Lett., 39, 3259, (1998).

11. S. A. Babirad, Y. Wang, Y. Kishi, J. Org. Chem. 52, 1370, (1987).

12. S. Jarosz, A. Zamqjski, J. Carbohydr. Chem., 12, 1223, (1993). 